\title{
Neurodegenerative diseases and their related treatment
}

\author{
Ziyan $\mathrm{Xin}^{1 *}$ \\ ${ }^{1}$ Beijing Neworiental Foreign Language School at Yangzhou, 225006 Yangzhou, Jiangsu, China
}

\begin{abstract}
Aging is a phenomenon related to every person and has a tremendous impact on people's life quality. During aging, some people may get neurodegenerative diseases, like Alzheimer's disease. These diseases can vastly impair patient's life quality and cause problems to their families. This review investigates and concludes many recent findings to find the symptoms, pathways, and possible cures for such diseases. The passage shows that changes happen at people's gene, special protein function, and brain structure are closely related to such diseases. Consistent with such opinions, this review concludes what symptoms patients may have, such as A $\beta$ 's accumulation and tau protein's hyperphosphorylation, degeneration of basal ganglia. This review also focuses on the possible ways to cure the diseases or release their symptoms. At last, in light of the knowledge we have about neurodegenerative diseases, a more comprehensive understanding of the critical parts involved in these diseases can be obtained, indicating possibilities of producing more closely targeted medicine and treatment.
\end{abstract}

\section{Introduction}

During normal aging, few people, but statistically significant, can get neurodegenerative diseases [1]. Such diseases are well known due to their significant fatal rate and inconvenience caused to patients and their relatives. As a result, neurodegenerative diseases have attracted a lot of attention from the public, and many researches have been conducted in this area. Scientists have already figured out that oxidative stress [2], change of epigenome [1], microglia cells' mitophagy [3], and a lot of other factors are closely related to such diseases. In this passage, the author wants to emphasize aspects about changes in neurological state and their relationship with many neurodegenerative diseases in which age is a risk factor, such as Alzheimer's disease (AD), Parkinson's disease (PD), and several forms of dementia.

\section{Aging symptoms and Neurodegenerative diseases}

Aging is a process that not only humans but also all living organisms cannot escape. A lot of physical changes can happen during this process, including mitochondrion dysfunction [4], muscle atrophy [5], NAD+'s decline in metabolism, and its caused energy deficiency [6], and damage of joints. Aging can involve various issues like DNA methylation [7], insulin imbalance [8], changes in genes [1], and the change in neuron synapses morphology [9]. Aging's effect on people's nervous system is tremendous [9]. During normal aging, a drastic decline of neuron number does not usually occur. Instead, small, region-specific changes in dendritic branching and spine density are more commonly observed, just like the thin spine loss in the prefrontal cortex and the reduction of post synaptic density area in hippocampal CA1 synapses. Such reduction will also result in a deficit of long-term potentiation (LTP) and more susceptibility to long-term depression (LTD) [9]. However, suppose people have some neurodegenerative diseases. In that case, one significant change that happens in their brains is the loss of large amounts of neurons, and finally leads to the severe impairment of cognitive and motive ability [10]. Such diseases mostly occur during the late period of people's lives, so understanding their symptoms' difference from the normal aging process is imperative. The loss of cognitive and motive ability [11] will cause a lot of inconveniences not only to patients themselves but also to their families. So, it is critical to understand the pathway behind such symptoms and what has neurodegenerative diseases changed.

\section{Alzheimer's disease (AD)}

\subsection{Symptoms of Alzheimer's disease}

In Alzheimer's disease (AD) and other neurodegenerative diseases, the most significant change is the death of neuronal cells. In Alzheimer's disease, patients will experience continuous neuronal death, which begins at the hippocampus [10]. They cannot control motion and have rudimentary reflection such as grabbing and sucking. Patients also experience significant aphasia [12]. Their cognitive reserve and cognition ability are severely damaged in the long term [13]. They also need to set posture for balance control [14]. As a result, patients are

\footnotetext{
* Corresponding author: shanchunjian@njmu.edu.cn
} 
enduring a series of changes that undermine their living quality.

\subsection{Pathways and physical changes related to AD}

\subsubsection{Physical changes}

During AD, atrophy of the middle temporal lobe can be observed by MRI. Similarly, the volume of the hippocampus, entorhinal cortex, and amygdala also shows signs of reduction. Hippocampus is related to long-term and short-term memory formation, although it may not be the place storing such memories. So, the atrophy of the hippocampus can account for patients' declined ability in normal and spatial memory. Amygdala is the place operating fear and anxiety, so its morphology can lead to abnormal emotional changes. Through PET test, the bilateral temporal-parietal lobe shows less glucose consumption, which is also evidence of the disfunction of these critical areas [15](figure1).

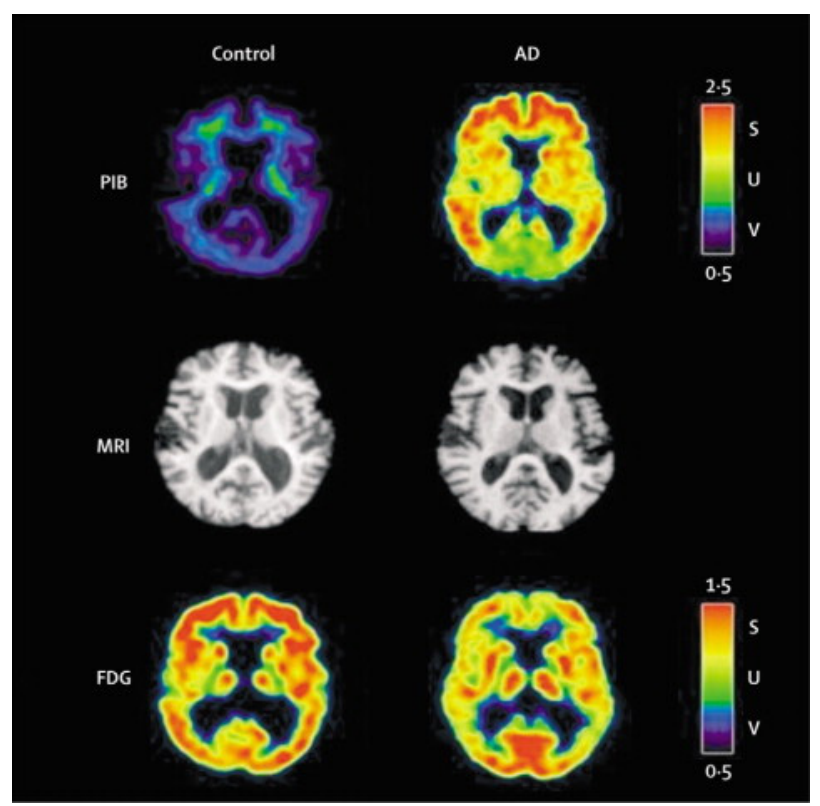

Fig 1. Comparison of PIB images, MRI, and FDG-PET images from a cognitively normal person and a patient with mild Alzheimer's disease

\subsubsection{Molecular biomarker}

The most distinct and significant thing that happens in AD patients might be the accumulation of $A \beta$ amyloid and the hyperphosphorylation of Tau protein. Amyloid - $\beta$ (a $\beta)$ is a polypeptide with 39-43 amino acids produced by amyloid precursor protein (APP) through the proteolysis of $\beta$ - and $\gamma$ - secretase. It can be produced by many kinds of cells and circulates in the blood, cerebrospinal fluid, and brain interstitial fluid (figure2). Most of them combine with chaperone molecules, and a few exist in the free state. The most common subtypes of a $\beta$ in the human body are $A \beta \quad 1-40$ and $A \beta$ 1-42. In human cerebrospinal fluid and blood, the content of a $\beta 1-40$ is 10 times and 1.5 times higher than that of $A \beta 1-42$, respectively. $A \beta 1-42$ is more toxic and easier to aggregate, thus forming the core of a $\beta$ precipitation and causing neurotoxicity. In 1991, Kowall et al. injected a $\beta$ into the cerebral cortex of rats or monkeys. They found that tissue necrosis, loss of peripheral neurons, and proliferation of neurokeratin occurred at the injection site, significantly correlated with the dose. The toxic effect of the nervous system is amyloidosis of the vascular wall, which directly leads to arteriosclerosis, poor elasticity, even easy rupture or thrombosis, and induces premature apoptosis of nerve cells. $A \beta$ thus can cause neurite withdrawal and neuronal degeneration [15]. On the other hand, Tau protein is a kind of microtubule-associated protein, and the microtubule system is a component of the neurocytoskeleton, which can participate in a variety of cell functions. Microtubules are composed of tubulin and microtubule-associated proteins. Since the Tau protein is the most abundant microtubule-associated protein, it is really important and plays a significant role in keeping neuronal configuration. The cellular function of tau protein in the normal brain is to combine with tubulin to promote its polymerization to form microtubules; to combine with microtubules to maintain the stability of microtubules, reduce the dissociation of tubulin molecules, and induce microtubule bundles. Tau protein is a phosphorylated protein. Tau protein in the normal mature brain contains 2-3 phosphorylated groups. Tau protein in the brain of Alzheimer's disease (AD) patients is abnormally hyperphosphorylated. Tau protein can contain 5-9 phosphate groups per molecule and lose its normal biological function. The binding ability of tau protein to tubulin is only $1 / 10$ of that of normal tau protein after abnormal hyperphosphorylation. It also loses its biological function of promoting microtubule assembly and maintaining microtubule stability. As a result, neurons may change their configuration and die [10]. Above is how these two important proteins affect neurons and cause neuronal disability. Besides, oxidative stress can account for the formation of Alzheimer's disease. Aging is associated with the generation and accumulation of reactive oxygen species (ROS) that are the major contributors to oxidative stress. Oxidative stress is caused by the imbalance between the production of ROS and their oxidation, which can affect the mitochondrial respiratory chain function, thereby altering the membrane permeability and calcium homeostasis and increasing the heteroplasmic mtDNA and weakening the mitochondrial defense systems [2]. Results in animal and cellular models of $\mathrm{AD}$ and in patients with sporadic late-onset $\mathrm{AD}$ suggest that impaired mitophagy contributes to synaptic dysfunction and cognitive deficits by triggering $A \beta$ and Tau accumulation through increases oxidative damage and cellular energy deficits; these, in turn, impair mitophagy [16]. 

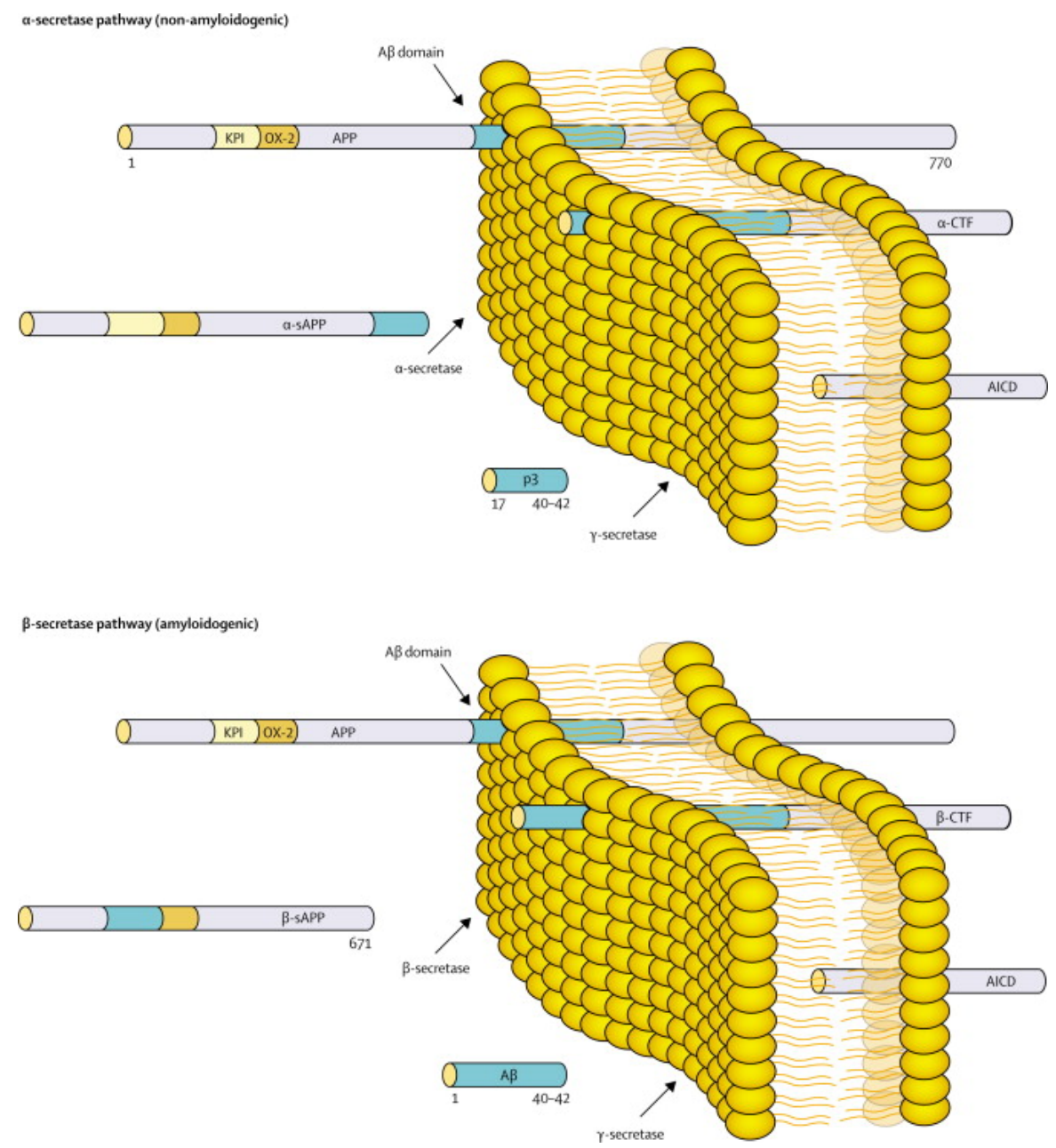

Fig 2. Metabolism of amyloid precursor protein (APP) with amyloid $\beta(A \beta)$ generation [32]

\subsection{Parkinson's diseases (PD) and Huntington's disease (HD)}

\subsubsection{PMA and SMA}

You are free to use colour illustrations for the online version of the proceedings. Still, any print version will be printed in black and white unless special arrangements have been made with the conference organiser. Please check with the conference organiser whether or not this is the case. If the print version is black and white only, you should check your figure captions carefully and remove any reference to colour in the illustration and text. Besides, some colour figures will degrade or suffer the loss of information when converted to black and white, which should be taken into account when preparing them.

In people's brains, there is a special pathway controlling motion. In this pathway, the primary motor cortex M1 (area 4) is responsible for moving. the parts related to $\mathrm{PD}$ and $\mathrm{HD}$ are different but also imperative to make a motion. According to researches, area 6, which consists of the premotor area (PMA) [17] and supplementary motor area (SMA) [18], is critical in forming voluntary movement. PMA connects with the neurons of the reticular spinal tract, which controls the motor units of the proximal muscles. The axons of SMA directly control the motor units of distal muscles. An experiment with monkey reveals that before motion, the PMA and SMA will first be activated, and then the M1 area will be activated. The loss of functioning in PMA and SMA will cause disability involuntary motion [17]. However, the symptom in PD and HD is totally opposite with one another, so there must be a thing can control these tow cortexes. Thus the different pathology in PD and HD will express different symptoms.

\subsubsection{Basal ganglia and its effect}

Basal ganglia are comprised of the caudate nucleus, putamen, globus pallidus, and subthalamic nucleus. Substantia nigra can also be ascribed to basal ganglia. This pathway can launch and screen voluntary movement. The caudate nucleus and putamen are called the striatum. The striatum is the source of input from the cortex to basal ganglia to the target nucleus. Simultaneously, the globus pallidus is the cephalic source of input from basal ganglia to thalamus. Basal ganglia's effect depends on the ventral lateral nucleus (VL), which is the main input to area 6 cortex is located in the dorsal thalamus. Basal ganglia are responsible for repressing VL. This pathway originates from the excitatory connection between cortex and putamen cells. The putamen cells formed inhibitory synapses with globus pallidus neurons, and the latter formed inhibitory synapses with VL neurons. The 
thalamic cortical link from VL to SMA is excitatory, enhancing the discharge of SMA motor-related neurons. The activation of cortical function by putamen via VL results in SMA excitation, so how does this happen. At rest, neurons in the globus pallidus spontaneously discharge, thus inhibiting VL. When the cortex is activated: 1 . The putamen neurons are excited; 2 . The putamen neurons inhibit the globus pallidus neurons; 3 . The VL neurons are released from the inhibition of the globus pallidus so that the VL neurons can start their activities, and the VL neurons promote SMA activities. Therefore, this loop functions as a positive feedback loop, which gathers or collects activities from a wide range of cortical areas into the auxiliary motor cortex. So when the signals gathered in the basal ganglia reach a certain threshold, the brain will send out signals for movement production [19].

\subsubsection{Phenotype of PD and HD}

The phenotype in PD mainly includes static tremors, bradykinesia, myotonia, and postural gait disorders. At the same time, patients may have depression, constipation, sleep disorders, and other non-motor symptoms [20]. The prominent pathological changes of Parkinson's disease are the degeneration and death of dopaminergic neurons in substantia nigra due to oxidative stress [21], the significant decrease of Da content in the striatum, and the appearance of eosinophilic inclusion bodies (Lewy body) in the cytoplasm of remaining neurons in substantia nigra [22]. The dopaminergic neurons in substantia nigra died more than $50 \%$, and the Da content in the striatum decreased more than $80 \%$. HD is a rare autosomal dominant disease. The patients usually have symptoms of movement, cognition, and spirit in middle age. The clinical symptoms of Huntington's disease are complex and changeable. The patient's condition is progressive and usually dies 15-20 years after onset. The main characteristics of the disease are chorea and dystonia with progressive cognition, mental dysfunction, and dementia. The cause is the wrong expression of the multiple nucleotides repeat in the Huntington gene, which affects different molecular pathways and eventually leads to neurological dysfunction and degeneration [23].

\subsubsection{Pathways that cause $P D$ and $H D$}

The pathogenesis of Parkinson's disease is based on the afferent pathway from substantia nigra to striatum to degeneration [21]. The neurotransmitter used by these afferent pathways is dopamine. Under normal conditions, the input from substantia nigra to striatum can liberate the ventrolateral nucleus (VL) from the inhibition of globus pallidus by activating putamen cells to enhance the activity of the direct motor loop. In essence, dopamine depletion blocks the input pathways to SMA through the basal ganglia and VL, greatly inhibiting and reducing voluntary movement [17]. The discovery of PD genes has led to the hypothesis that misfolding of proteins and dysfunction of the ubiquitin-proteasome pathway are pivotal to PD pathogenesis. Previously implicated culprits in PD neurodegeneration, mitochondrial dysfunction, and oxidative stress may also act in part by causing the accumulation of misfolded proteins, in addition to producing other deleterious events in dopaminergic neurons. Neurotoxin-based models (particularly MPTP) have been important in elucidating the molecular cascade of cell death in dopaminergic neurons. PD models based on manipulating PD genes should prove valuable in elucidating important aspects of the disease, such as the selective vulnerability of substantia nigra dopaminergic neurons to the degenerative process [24]. If Parkinson's disease is one extreme of basal ganglia disease, Huntington's disease is the other. The disease is characterized by increased motor function, dyskinesia, or abnormal movement. The patient also shows chorea, which means that the patient has uncontrollable aimless movement. The most obvious lesion in Huntington's disease is the death of neurons in the caudate nucleus, putamen, and globus pallidus, accompanied by the death of neurons in the cerebral cortex and other parts. Therefore, the inhibitory ability of basal ganglia to SMA is greatly reduced, leading to aimless movement [18]. The abnormal place of Huntington's disease is htt protein. Normal htt protein itself has various cellular functions, and htt protein mutation leads to these dysfunctions. Protein variation is often the first manifestation of abnormal expression of related genes. Previous studies have shown that abnormal expression of genes related to nerve conduction in HD striatum. In addition, the abnormal repeat of CAG can affect the interaction between molecules on a large scale, leading to the disorder of intracellular protein transport. Htt protein variation not only disrupts the gene regulation of mitochondrial function related proteins but also reacts with proteins on the surface of the mitochondrial membrane, impairs the function of the respiratory chain, hinders the fixation of mitochondria to microtubules, affects the dynamic fusion and division of mitochondria, and increases calcium transport. The mutant protein can also inhibit autophagy, promote apoptosis, change neurotrophic energy supply and intracellular biological and signal synthesis [25].

\section{Treatment}

\subsection{Treatment to AD}

\subsubsection{The purpose of symptomatic treatment is to control the accompanying psychopathological symptoms}

Short-acting benzodiazepines, such as alprazolam, oxazepam, lorazepam, and triazolam, can be considered for anti-anxiety drugs with symptoms of anxiety, agitation, and insomnia. The dosage should be small and should not be used for a long time. The side effects such as excessive sedation, drowsiness, unclear speech, ataxia, and gait instability should be noted. About $20 \%-50 \%$ of AD patients with antidepressants have depressive symptoms. The patients with mild and short duration of depression 
should be given persuasion, psychotherapy, social support, and environmental improvement. Antidepressants can be added when necessary.

Antipsychotics help to control the patient's behavior disorder, agitation, aggression, hallucination, and delusion. However, a small dose should be used, and the drug should be stopped in time to prevent the occurrence of toxic and side effects. A small dose of perphenazine may be taken orally. The hypotension and extrapyramidal side effects of thioridazine are lighter than chlorpromazine. Thioridazine is one of the commonly used antipsychotics for the elderly

\subsubsection{Intelligence medicine or medicine for improving cognitive function}

The block of the cholinergic system, which acts on neurotransmitters, can cause the decline of memory and learning, similar to amnesia in normal elderly. If we strengthen the central cholinergic activity, we can improve the learning and memory ability of the elderly. Therefore, the change of the cholinergic system is closely related to the degree of cognitive impairment in $\mathrm{AD}$. The main functions of this kind of drugs are to expand cerebral blood vessels, increase the utilization of oxygen, glucose, amino acids, and phospholipids by cerebral cortex cells, promote the recovery of brain cells, improve the function of brain cells, so as to achieve the purpose of improving memory [26].

\subsubsection{Physical exercise may help improve patients' cognitive ability}

Meta-analysis and systematic review indicated that exercise intervention might improve the cognitive function of $\mathrm{AD}$ or slow down the decline of cognition; however, this relationship was not always true across studies [27].

\subsection{Treatment to PD}

\subsubsection{Drug treatment}

Anticholinergic drugs: mainly by inhibiting the activity of acetylcholine in the brain, the corresponding increase in dopamine effect (table1). Amantadine: it can promote the synthesis and release of dopamine in nerve endings and prevent its reabsorption. It may be effective for dyskinesia. Monoamine oxidase B (MAO-B) inhibitor: by irreversibly inhibiting MAO-B in the brain, blocking the degradation of dopamine, relatively increasing the content of dopamine to achieve the purpose of treatment. MAO-B inhibitor can be used as a single drug in the treatment of new onset and young patients with Parkinson's disease. It can also assist compound levodopa in the treatment of advanced patients. It may have a neuroprotective effect, so early use is recommended in principle. DR agonist: can directly stimulate dopamine receptors and play a role. Currently, non-ergot Dr agonists are commonly used in the clinic. It can also be used in combination with compound levodopa in the treatment of advanced patients. MAO-B inhibitor or Dr agonist is the first choice in young patients. Compound levodopa (including levodopa / Benserazide and levodopa/carbidopa): levodopa is the precursor of dopamine. Peripheral levodopa can be converted into dopamine by decarboxylation of dopa decarboxylase in the brain through the blood-brain barrier so as to play the role of alternative therapy. Benserazide and carbidopa are inhibitors of peripheral decarboxylase, which can reduce L-dopa's decarboxylation in peripheral blood and increase the content of L-dopa in the brain and reduce its peripheral side effects [28].

Table1. Investigational anti-Alzheimer's drugs.

\begin{tabular}{lllll}
\hline Target & Drug & Study phase & Expected completion date & Results \\
\hline -Amyloid & CAD106 & 2 & May 2024 & \\
& CNP520 & 2 & May 2024 & \\
BAN2401 & 2 & November 2018 & \\
& LY3002813* & 2 & December 2020 & \\
Crenezumab & 3 & October 2022 & Not effective \\
Aducanumab & 3 & April 2022 & Safe for phase 3 \\
UB-311 & 2 & December 2018 & \\
Gantenerumab & 3 & November 2019 & \\
Solanezumab & 3 & Terminated & \\
& CT1812 & 2 & May 2017 & \\
& & October 2016 & \\
Thiethylperazine & 2 & July 2021 & \\
ID1201 & 2 & December 2018 & \\
NPT088 & 1 & February 2019 & \\
Lu AF20513 & 1 & October 2018 & \\
ABvac40 & 2 & February 2021 & \\
\hline
\end{tabular}




\begin{tabular}{|c|c|c|c|c|}
\hline & Ponezumab & 2 & $\begin{array}{l}\text { Completed } \\
\text { June } 2011\end{array}$ & Not effective \\
\hline & ACC-001 & 2 & $\begin{array}{l}\text { Completed } \\
\text { February } 2014\end{array}$ & Safe for phase 3 \\
\hline & KHK6640 & 1 & $\begin{array}{l}\text { Completed } \\
\text { December } 2017\end{array}$ & None yet \\
\hline & GSK933776 & 2 & Completed & Not effective \\
\hline & UB-311 & 1 & Completed & Safe for phase 2 \\
\hline & ABvac40 & 1 & $\begin{array}{l}\text { Completed } \\
\text { July } 2015\end{array}$ & Safe for phase 2 \\
\hline \multirow[t]{6}{*}{ BACE1 } & Lanabecestat & 2 & September 2019 & \\
\hline & JNJ-54861911 & 2 & October 2022 & \\
\hline & Elenbecestat & 3 & December 2020 & \\
\hline & LY3202626* & 2 & December 2020 & \\
\hline & Verubecestat & 3 & March 2021 & \\
\hline & LY450139 & 3 & $\begin{array}{l}\text { Completed } \\
\text { April } 2011\end{array}$ & Not effective \\
\hline \multirow[t]{10}{*}{ P-tau } & $\begin{array}{l}\text { IONIS- } \\
\text { MAPTRx }\end{array}$ & 1,2 & February 2020 & \\
\hline & JNJ-63733657 & 1 & February 2019 & \\
\hline & RO7105705 & 2 & September 2022 & \\
\hline & ABBV-8E12 & 2 & June 2021 & \\
\hline & AADvac1 & 2 & June 2019 & \\
\hline & BIIB-092 & 2 & September 2020 & \\
\hline & BIIB-080 & 1 & February 2020 & \\
\hline & TPI-287 & 1 & $\begin{array}{l}\text { Completed } \\
\text { May } 2017\end{array}$ & \\
\hline & $\mathrm{TRx} 0237$ & 3 & February 2019 & \\
\hline & LY3303560 & 1 & June 2019 & \\
\hline APP & Posiphen & 1 & & \\
\hline RAGE & Azeliragon & 3 & $\begin{array}{l}\text { Terminated } \\
\text { January } 2019\end{array}$ & Not effective \\
\hline \multirow[t]{2}{*}{ Retinoid receptor } & Acitretin & 2 & $\begin{array}{l}\text { Completed } \\
\text { February } 2018\end{array}$ & \\
\hline & Bexarotene & 2 & $\begin{array}{l}\text { Completed } \\
\text { February } 2016\end{array}$ & \\
\hline
\end{tabular}

Potential treatments currently undergoing clinical investigation. APP, amyloid precursor protein; BACE1, -site amyloid precursor protein cleaving enzyme 1; p-tau, hyperphosphorylated tau peptide; RAGE, receptor for advanced glycation end products.

*Medications under investigation as combination therapy. Source: www.clinicaltrials.gov.

\subsubsection{Non-drug treatment}

There are mainly two kinds of operation methods, neuronucleolysis and deep brain stimulation (DBS). The common targets for neuronucleolysis are the ventral intermediate nucleus of the thalamus (VIM) and the ventral posterior pallidum (PVP). The ventral intermediate nucleus of the thalamus was selected for the patients with tremors, and the ventral posterior pallidum was selected for the patients with rigidity. Because of its low cost and certain curative effect, it is still used in some places. Deep brain stimulation has been the first choice of surgical treatment because of its minimally invasive, safe, and effective. Patients with Parkinson's disease have obvious curative effect decline or dyskinesia, who cannot improve the symptoms well after drug adjustment can consider surgical treatment. The effect of the operation on limb tremor and myotonia is good. Still, there is no significant improvement on axial symptoms such as abnormal posture and gait, dysphagia, and so on. Surgery, like drug therapy, can only improve symptoms but cannot cure the disease or prevent the progress of the disease [29]. It is necessary to take medicine after the operation, but the dosage can be reduced. The surgical treatment of secondary parkinsonism and parkinsonism plus syndrome was ineffective. Early Parkinson's disease patients with good drug treatment effects are not suitable for early surgery. Methods of mental relaxation and auditory training, methods of whole-body vibration (vibromassage), laser therapy (photoacoustic therapy), acupuncture may provide alternative solutions in this situation [30]. 


\subsection{Treatment to HD}

Due to the lack of research on drug treatment of cognitive impairment in HD, there is still no effective drug. For obsessive-compulsive and irritable symptoms, stepwise treatment is recommended. In patients with mild cognitive impairment, serotonin reuptake inhibitors can be used first, combined with behavioral therapy. If depression, anxiety, and obsessive-compulsive behaviors occur simultaneously, serotonin reuptake inhibitors can also be used as first-line drugs. When the dosage is adjusted to the optimum, it should be combined with medication. Behavior therapy can also be used. Dysphagia usually occurs in the later stage of the disease. At present, there are guidelines for swallowing food (guidance should be started before cognitive impairment affects learning ability), including correct food preparation and food provision under a monitoring environment. It is very important to increase the energy intake of patients with appropriate methods. There is no specific and effective treatment for Huntington's chorea. Some drugs can relieve symptoms but can't stop the disease from progressing. Etiological treatment is carried out by gene level or cell level, but both are in the research stage. Because of its unique advantages, deep brain stimulation has been applied to patients with the relatively rapid progress of motor symptoms. The target of electrical stimulation is mainly located in the medial part of bilateral globus pallidus. Studies have found that electrical stimulation can improve the motor symptoms of HD patients and improve the mood, physical strength, and activities of daily living. Therefore, the improvement of motor function can significantly improve the quality of life and prolong the time of independent life in patients with mild involvement of higher cortical function. As a new therapeutic method, gene therapy, especially RNAi and neurotrophic factor gene transfer, has shown certain efficacy in Huntington's disease animal model. However, it is still a long way from animal experiments to human experiments. HD patients and their relatives will also face many social and medical problems, which need to be reasonably solved by trained professionals $[25,31]$.

\section{Conclusion}

In summary, instead of subtle changes in neuronal morphology, synaptic integrity, cellular connectivity, and gene expression, patients experience the death of neuron cell body during neurodegenerative diseases. A decrease of hippocampal and basal ganglia's function can impair patients' cognitive and motive ability. Evidence points out that accumulation of $A \beta$ and the hyperphosphorylation of tau protein are associated with neuron death in Alzheimer's disease. Denaturing of basal ganglia can cause over-excited or repressed voluntary movement ability. Related methods of curing such diseases include behavior and medical treatment. However, the medicine used for curing is not fully understood, and its target is not always clear, so the medicine's side effect is not clear, and its functioning pathway is not fully understood. Future work in neurodegenerative diseases should focus on the whole pathway of cognitive and motive behavior, guiding the medicine research. It is possible that future medicine can slow down the progress of neurodegenerative diseases and even reverse the symptoms after fully understanding what is happening in patients' brains.

\section{References}

1. Hou, Y., et al., Ageing as a risk factor for neurodegenerative disease. Nat Rev Neurol, 2019. 15(10): p. 565-581.

2. Elfawy, H.A. and B. Das, Crosstalk between mitochondrial dysfunction, oxidative stress, and age related neurodegenerative disease: Etiologies and therapeutic strategies. Life Sci, 2019. 218: p. 165-184

3. Perry, V.H., J.A. Nicoll, and C. Holmes, Microglia in neurodegenerative disease. Nat Rev Neurol, 2010. 6(4): p. 193-201.

4. Kauppila, T.E.S., J.H.K. Kauppila, and N.G. Larsson, Mammalian Mitochondria and Aging: An Update. Cell Metab, 2017. 25(1): p. 57-71.

5. Lacolley, P., et al., Vascular Smooth Muscle Cells and Arterial Stiffening: Relevance in Development, Aging, and Disease. Physiol Rev, 2017. 97(4): p. 1555-1617.

6. Imai, S. and L. Guarente, NAD+ and sirtuins in aging and disease. Trends Cell Biol, 2014. 24(8): p. 464-71.

7. Jung, M. and G.P. Pfeifer, Aging and DNA methylation. BMC Biol, 2015. 13: p. 7.

8. Sasako, T. and K. Ueki, [Insulin/IGF-1 signaling and aging]. Nihon Rinsho, 2016. 74(9): p. 1435-1440.

9. Shankar, S.K., Biology of aging brain. Indian J Pathol Microbiol, 2010. 53(4): p. 595-604.

10. Chi, H., H.Y. Chang, and T.K. Sang, Neuronal Cell Death Mechanisms in Major Neurodegenerative Diseases. Int J Mol Sci, 2018. 19(10).

11. Ding, W., et al., Neurodegeneration and cognition in Parkinson's disease: a review. Eur Rev Med Pharmacol Sci, 2015. 19(12): p. 2275-81.

12. Shinagawa, S., [Language Symptoms of Alzheimer's Disease]. Brain Nerve, 2016. 68(5): p. 551-7.

13. Soldan, A., et al., Cognitive reserve and long-term change in cognition in aging and preclinical Alzheimer's disease. Neurobiol Aging, 2017. 60: p. 164-172.

14. Chong, R.K., C.L. Jones, and F.B. Horak, Postural set for balance control is normal in Alzheimer's but not in Parkinson's disease. J Gerontol A Biol Sci Med Sci, 1999. 54(3): p. M129-35.

15. Sengoku, R., Aging and Alzheimer's disease pathology. Neuropathology, 2020. 40(1): p. 22-29.

16. Kerr, J.S., et al., Mitophagy and Alzheimer's Disease: Cellular and Molecular Mechanisms. Trends Neurosci, 2017. 40(3): p. 151-166.

17. Li, N., et al., Robust neuronal dynamics in premotor cortex during motor planning. Nature, 2016. 532(7600): p. 459-64. 
18. Nachev, P., C. Kennard, and M. Husain, Functional role of the supplementary and pre-supplementary motor areas. Nat Rev Neurosci, 2008. 9(11): p. 85669.

19. Albin, R.L., A.B. Young, and J.B. Penney, The functional anatomy of basal ganglia disorders. Trends Neurosci, 1989. 12(10): p. 366-75.

20. Jankovic, J., Parkinson's disease: clinical features and diagnosis. J Neurol Neurosurg Psychiatry, 2008. 79(4): p. 368-76.

21. Trist, B.G., D.J. Hare, and K.L. Double, Oxidative stress in the aging substantia nigra and the etiology of Parkinson's disease. Aging Cell, 2019. 18(6): p. e13031.

22. Wakabayashi, K., et al., The Lewy body in Parkinson's disease and related neurodegenerative disorders. Mol Neurobiol, 2013. 47(2): p. 495-508.

23. Walker, F.O., Huntington's disease. Lancet, 2007. 369(9557): p. 218-28.

24. Dauer, W. and S. Przedborski, Parkinson's disease: mechanisms and models. Neuron, 2003. 39(6): p. 889-909.

25. Ross, C.A. and S.J. Tabrizi, Huntington's disease: from molecular pathogenesis to clinical treatment. Lancet Neurol, 2011. 10(1): p. 83-98.

26. Briggs, R., S.P. Kennelly, and D. O’Neill, Drug treatments in Alzheimer's disease. Clin Med (Lond), 2016. 16(3): p. 247-53.

27. Du, Z., et al., Physical activity can improve cognition in patients with Alzheimer's disease: a systematic review and meta-analysis of randomized controlled trials. Clin Interv Aging, 2018. 13: p. 1593-1603.

28. Armstrong, M.J. and M.S. Okun, Diagnosis and Treatment of Parkinson Disease: A Review. Jama, 2020. 323(6): p. 548-560.

29. Habets, J.G.V., et al., An update on adaptive deep brain stimulation in Parkinson's disease. Mov Disord, 2018. 33(12): p. 1834-1843.

30. Pokhabov, D.V., V.G. Abramov, and D.D. Pokhabov, [Possibilities of non-drug treatment for Parkinson's disease]. Zh Nevrol Psikhiatr Im S S Korsakova, 2016. 116(8): p. 22-29.

31. Wyant, K.J., A.J. Ridder, and P. Dayalu, Huntington's Disease-Update on Treatments. Curr Neurol Neurosci Rep, 2017. 17(4): p. 33.

32. Blennow K, Leon M, Zetterberg H . Alzheimer's disease.[J]. Lancet, 2006, 368(9533):387-403. 